\title{
Editorial
}

Ocular Oncology

and Pathology
Ocul Oncol Pathol 2017;3:I-III

DOI: 10.1159/000453361
Received: November 7, 2016

Accepted: November 10, 2016

Published online: December 24, 2016

\section{Advancing the Treatments of Retinoblastoma: Stuck in the 1950s}

\author{
Matthew W. Wilson \\ Department of Ophthalmology, Hamilton Eye Institute, University of Tennessee Health Science Center, and \\ Departments of Surgery and Pathology, St. Jude Children's Research Hospital, Memphis, TN, USA
}

With advancing age and wisdom, we are often apt to romance the simpler time of our youth. The world was less complicated. We were not hyperstimulated in an era devoid of email, cell phones, and texts. However, survival rates from pediatric cancers, notably retinoblastoma, were much lower. There was no Knudson two-hit hypothesis, RB1 gene, patient-derived orthotopic xenografts, or whole-genome sequencing. However, there was external beam radiation [1], intra-arterial therapy [2], intravitreal therapy [3], and melphalan [4]. Fast-forward to the present, where advances in science and medicine are improving health care, but with the background force of social media. Our challenge as clinicians and scientists is to assure that science and medicine are kept in parallel with bench-to-bedside research as a feedback loop. The medical treatment we bring to our patients today must be thoroughly evaluated using the research tools that have been made available.

In this issue of Ocular Oncology and Pathology, we are presented with 2 separate case reports, each documenting the novel use of intracameral melphalan for the treatment of anterior chamber seeding from advanced intraocular retinoblastoma $[5,6]$. The first patient was an 11-year-old with unilateral International Classification of Retinoblastoma (ICRB) Group E eye with anterior chamber seeding and involvement of the trabecular meshwork for 270 degrees. The visual acuity was "normal OU". Initial treatment consisted of a multitier approach using intra-arterial, intravitreal, and intracameral melphalan. This was followed by 4 monthly cycles of neoadjuvant systemic carboplatin and etoposide. An anterior chamber recurrence was noted 3.5 months later and further intracameral and intravitreal melphalan were delivered. Reported complications included heterochromia and opacification of the lens cortex, necessitating cataract surgery. Endothelial cell density remained stable during therapy; however, following cataract surgery, a $10 \%$ endothelial cell loss was noted. At 5 years of follow-up, the child was disease free with 20/20 vision in both eyes. The second patient had bilateral retinoblastoma (ICRB Group D right eye and Group E left eye) diagnosed at 1 year of age. The left eye had been enucleated, and the right eye developed ciliary body and anterior chamber relapse. Intraophthalmic artery chemotherapy was ineffective, so in hopes of avoiding external radiation, the authors elected to couple 4 alternating courses of intravitreal and intracameral melphalan with a seventh cycle of systemic chemotherapy. At 3 years of follow-up, the patient had no recurrent disease and "useful vision" despite documented atrophic choroidal scarring and retinal vascular toxicity.

\section{KARGER}

(c) 2016 S. Karger AG, Basel

E-Mail karger@karger.com

www.karger.com/oop
Matthew W. Wilson, MD, FACS

Department of Ophthalmology, Hamilton Eye Institute, University of Tennessee Health Science Center, 930 Madison Avenue

Memphis, TN 38163 (USA)

mwilson@uthsc.edu 
Intracameral melphalan builds upon a decade of local chemotherapy delivery methods for the treatment of intraocular retinoblastoma. We have moved rapidly from periocular to intra-arterial to intravitreal and now to intracameral administration with the specific aims of delivering an increasingly higher concentration of chemotherapy in hopes of controlling ocular disease, while mitigating systemic side effects [7-9]. These advances have been heralded as successes, but each in turn has been overshadowed by a battery of subsequent case reports and small case series detailing complications of the technique [1013]. Many reports show late toxicity to the organ for which salvage was attempted, while reports of metastatic disease remind us that losing sight of the entire child can have dire consequences. Such has been the history of retinoblastoma management from the era of external beam radiotherapy through systemic chemotherapy to the present. However, the rapidity at which we cycle through therapies has accelerated, leaving little time to assess the balance of efficacy and toxicity. In the end, as clinicians, we are left with a paucity of evidence-based medicine to improve outcomes in our retinoblastoma patients.

Central to this argument is that any treatment we render does leave a proverbial footprint upon our vulnerable patient population [14]. Late effects is a fertile research environment in pediatric oncology but one that often seems overlooked in retinoblastoma. There was a 50-year lag between the adoption of external beam radiotherapy as a standard-of-care therapeutic approach to retinoblastoma patients and the reported increased incidence of second malignancies in retinoblastoma survivors treated with this modality [15]. To prevent such treatment-related morbidity, it is imperative that we understand what we are doing prior to doing it. We have designed a decade worth of novel approaches to therapy in a void of pharmacology and pharmacokinetics, all the while citing a single in vitro cell toxicity assay to legitimize our choice of a single chemotherapeutic agent developed in the 1950s as a derivative of nitrogen mustard [16]. Our vision has been laser focused on local delivery, but horribly myopic in our choice of a drug.

The overall survival rate for pediatric cancers has improved from approximately $30 \%$ in the 1970 s to over $80 \%$ currently, and retinoblastoma survival rates now exceed $90 \%$ in developed countries. For pediatric malignancies, this improvement is undeniably linked to the enrollment and monitoring of patients on prospective clinical protocols that have helped stratify patients according to clinical and genomic risk features, optimize treatments, and attenuate long-term morbidities [17]. While clinical trials have been essential in advancing the field of pediatric oncology, there is a significant time lapse from trial design to implementation to outcome data. To keep the science of tumor biology, technological advances, and treatment in parallel, we must rely upon preclinical models that incorporate the disciplines of chemical biology and pharmacology. Bench-to-bedside medicine can lead to prospective, retinoblastoma protocol-driven research studies with reportable patient outcomes, toxicities, and improved ocular event-free survival with long-term vision data [18]. These results are generalizable to the retinoblastoma community, rather than specific to a single patient on a given day in a certain clinic. Bench-to-bedside medicine has also identified novel targeted pathways for drug development and prevented us from subjecting our patients to unnecessary toxicities $[19,20]$. None of the treatment modalities discussed in these 2 case reports was tested in a preclinical model with subsequent scientific peer review before being introduced into children. So for the time being, we must await the barrage of case reports and small case series that will come forth, heralding successes or horrors, while we continue to be stuck in the 1950s.

\section{References}

1 Reese AB, Merrian GR Jr, Martin HE: Treatment of bilateral retinoblastoma by irradiation and surgery: report of 15-years results. Am J Ophthalmol 1949;32:175-190.

2 Reese AB, Hyman GA, Tapley ND, Forrest AW: Treatment of retinoblastoma by radiation and triethylene melamine. AMA Arch Ophthalmol 1958;60:897-906.

3 Ericson L, Rosengren BHO: Present therapeutic resources in retinoblastoma. Acta Ophthalmol (Copenh) 1961;39:569-576.
4 Papac R, Galton DA, Till M, Wiltshaw E: Preliminary clinical trial of p-di-2-chloroethylamino-L-phenylalanine (CB 3025.mephalan) and of di-2-chloroethymetahnesulfonate (CB 1506). Ann NY Acad Sci 1958;68:1126-1127.

5 Munier FL, Gaillard M, Decembrini S, et al: Intracameral chemotherapy (melphalan) for aqueous seeding in retinoblastoma: a bicameral injection technique and related toxicity in a pilot case study. Ocul Oncol Pathol 2017;3:149-155.
6 Cassoux N: Eye salvage with combination of intravitreal and intracameral melphalan injection for recurrent retinoblastoma with anterior chamber involvement: report of a case. Ocul Oncol Pathol 2017;3:129-132.

7 Abramson DH, Frank CM, Dunkel IJ: A phase I/II study of subconjunctival carboplatin for intraocular retinoblastoma. Ophthalmology 1999;106:1947-1950.
II

Ocul Oncol Pathol 2017;3:I-III DOI: $10.1159 / 000453361$
Wilson 
8 Abramson DH, Dunkel IJ, Brodie SE, et al: A phase I/II study of direct intraarterial (ophthalmic artery) chemotherapy with melphalan for intraocular retinoblastoma: initial results. Ophthalmology 2008;115;1398-1404.

9 Munier FL, Gaillard M, Balmer A, et al: Intravitreal chemotherapy for vitreous disease in retinoblastoma revisited; from prohibition to conditional indications. $\mathrm{Br} \mathrm{J}$ Ophthalmol 2012;96:1078-1083.

10 Abramson DH: Periocular chemotherapy for retinoblastoma success with problems? Arch Ophthalmol 2005;123:128-129.

11 Yousef Y, Sameh SE, Paulta P, et al: Intra-arterial chemotherapy for retinoblastoma a systematic review. JAMA Ophthalmol 2016;134: 584-591.
12 Chao A, Kao LY, Liu L, Wang NK: Diffuse chorioretinal atrophy after a single standard low-dose intravitreal melphalan injection in a child with retinoblastoma: a case report. BMC Ophthalmol 2016;16:27.

13 Francis JH, Marr BP, Brodie SE, Abramson DH: Anterior ocular toxicity of intravitreous melphalan for retinoblastoma. JAMA Ophthalmol 2015;133:1459-1463.

14 Hudson MM, Ness KK, Gurney JG, et al: Clinical ascertainment of health outcomes among adults treated for childhood cancer. JAMA 2013;309:2371-2381.

15 Forrest AW: Tumors following radiation about the eye. Trans Am Acad Ophthalmol Otolaryngol 1961;65:694-717.

16 Inomata M, Kaneko A: Chemosensitivity profiles and cultured human retinoblastoma cells in a human tumor clonogenic assay. Jpn J Cancer Res 1987;78:858-868.
17 Brennan R, Federico S, Dyer MA: The war on cancer: have we won the battle but lost the war? Oncotarget 2010;1:77-83.

18 Brennan RC, Qaddoumi I, Mao S, et al: Ocular salvage and vision preservation using a topotecan-based regimen for advanced intraocular retinoblastoma. J Clin Oncol 2016, Epub ahead of print.

19 Brennan R, Federico S, Bradley C, et al: Targeting the p53 pathway in retinoblastoma with subconjunctival nutlin-3a. Cancer Res 2011;71:4205-4213.

20 Zhang J, Benavente CA, McEvoy J, et al: A novel retinoblastoma therapy from genomic and epigenetic analyses. Nature 2012;481: 329-334. 